\section{AB1280-HPR REQUIRED FORCE TO OBTAIN A POSITIVE SQUEEZE TEST AUTOMATIZED IN PATIENTS WITH HAND ARTHRALGIA}

M. M. Castañeda-Martínez ${ }^{1}$, G. Figueroa-Parra ${ }^{1}$, D. Vega-Morales ${ }^{1}$, B. R. Vázquez Fuentes ${ }^{1}$, Y. G. Ordoñez Azuara ${ }^{2}$, J. A. Esquivel Valerio ${ }^{1}$, D. Á. Galarza-Delgado'. 'University Hospital "Dr. José Eleuterio González", Rheumatology Service, Monterrey, Mexico; ${ }^{2}$ University Hospital "Dr. José Eleuterio González", Family Medicine Service, Monterrey, Mexico

Background: Primary care physicians (PCP) are usually the first contact of people with inflammatory rheumatic diseases, and find the early symptoms of Rheumatoid Arthritis (RA) difficult to distinguish from those of other rheumatic diseases. A time-delay in the reference to Rheumatology is a health issue in several countries. The clinical aspects that general practitioner took into account in hand arthralgia patients are important to make the reference. In particular the Squeeze Test (ST) - which is simple to perform and rapidly done, ST is useful for identifying progression to RA in patients with undifferentiated arthritis. The ST has been described as not reliable because is clinician-dependent.

Objectives: To identify the required force that needs to be applied in order to obtain a positive Automatized Squeeze Test (AST) in a cohort of patients with hand arthralgia.

Methods: Ninety-seven patients were recruited in Family Medicine Consultation and in Rheumatology Consultation of the Hospital Universitario "Dr. José Eleuterio González" in Monterrey, Nuevo León, México. Eligible patients were adults (aged $\geq 18$ years) with hand arthralgia (that wasn't caused by trauma) as their chief complaint. After obtaining informed consent and after a questionnaire application, patients were submitted to AST maneuver, using an automated compressor with different forces already predetermined in the interface of the software used for compression.

Results: In this cohort of 98 patients, $79(80.6 \%)$ were women. The mean age was 51.14 years (SD 14.66). Ninety-six (97.9\%) patients were right handed. The diagnoses were Osteoarthritis (OA) $(16.3 \%)$, RA (5.1\%), Undifferentiated arthritis (1.2\%), Psoriatic arthritis (1.2\%) and Fibromyalgia (2\%). Force measures according to diagnoses are reported in Table 1.

Table 1. Diagnoses and mean forces

\begin{tabular}{lccc}
\hline Diagnosis & $\mathbf{n}(\%)$ & $\begin{array}{c}\text { Right hand force mean } \\
\mathbf{( k g / \mathbf { s } ^ { 2 } )} \mathbf{( S D )}\end{array}$ & $\begin{array}{c}\text { Left hand } \\
\text { force mean } \\
\mathbf{( k g / \mathbf { s } ^ { 2 } )}(\mathbf{S D})\end{array}$ \\
\hline OA & $16(16.3)$ & $3.53(2.74)$ & $3.18(2.73)$ \\
RA & $5(5.1)$ & $3.60(2.53)$ & $3.16(1.36)$ \\
UA & $1(1.2)$ & $7.60(0)$ & $8.70(0)$ \\
PsA & $1(1.2)$ & $7.60(0)$ & $7.80(0)$ \\
FM & $2(2.0)$ & $4.11(4.40)$ & $1.75(1.06)$ \\
\hline
\end{tabular}

OA, Osteoarthritis; RA, Rheumatoid Arthritis; UA, Undifferentiated Arthritis; PsA, Psoriatic Arthritis; FM, Fibromyalgia; SD, Standard Deviation

Conclusion: In the cases of RA and OA, the means of force to obtain a positive AST was lower than in the rest of the diagnoses.

References:

[1] Stack R, Nightingale P, Jinks C, Shaw K, Herron-Marx S, Horne R et al. Delays between the onset of symptoms and first rheumatology consultation in patients with rheumatoid arthritis in the UK: an observational study. BMJ Open. 2019;9(3):e024361.

Disclosure of Interests: None declared

DOI: 10.1136/annrheumdis-2020-eular.4515

\section{AB1281-HPR THE LEVELS OF VITAMIN D IN THE SPONDYLOARTHRITIS. DOES THE DEFICIT CORRESPOND TO THE INFLAMMATORY ACTIVITY?}

D. Castro-Corredor ${ }^{1}$, M. A. Ramírez Huaranga ${ }^{2}$, A. I. Rebollo Giménez ${ }^{2}$, M. D. Mínguez Sánchez ${ }^{2}$, J. Anino-Fernández ${ }^{2}$, I. M. De Lara Simón ${ }^{3}$, J. L. Cuadra Díaz ${ }^{2}{ }^{1}$ Hospital General Universitario Ciudad Real, Ciudad Real, Spain; ${ }^{2}$ Hospital General Universitario Ciudad Real, Rheumatology Department, Ciudad Real, Spain; ${ }^{3}$ Hospital General Universitario Ciudad Real, Pathology Department, Ciudad Real, Spain

Background: Spondyloarthritis is a group of chronic inflammatory diseases with involvement of the axial skeleton (mainly), and also of peripheral joints. Patients with spondyloarthritis have a significant prevalence of vitamin $\mathrm{D}$ levels below normal and that would correlate with the degree of activity of the disease.
Objectives: To determine the association between vitamin $D$ deficiency and the degree of activity of the disease (inflammatory activity) in a cohort of patients with spondyloarthritis.

Methods: Case-control type analytical observational study. We propose a retrospective review of the database of patients with spondyloarthritis (according ASAS2010 criteria) who were treated in the outpatient clinics of the Rheumatology Service of the General University Hospital of Ciudad Real during June 2018 to June 2019. Patients with the data will be selected. necessary for the analysis of the variables under study. The numerical variables of normal distribution evaluated will be described using measures of frequency and measures of central tendency / dispersion as appropriate. To assess the association between vitamin $\mathrm{D}$ levels and activity index, the odds ratio (OR) is calculated, with a $95 \%$ confidence level and the T-student for related samples.

Results: The final results of the study are presented. 115 patients were analyzed, of which 64 were men and 51 women, with an average age of 45.97 years (+/- 13.41 DE). $47 \%$ were ankylosing spondylitis, $21 \%$ psoriatic arthropathy, $16 \%$ undifferentiated spondyloarthritis, $7 \%$ spondyloarthropathy associated with inflammatory bowel disease and $9 \%$ were spondyloarthropathy associated with inflammatory bowel disease. The average of the activity was a BASDAI of 4.57 (+/- 2.35 SD) and measured by DAPSA was 12.61 (+/- 6.76 SD). 63 and 14 patients had activity measured by BASDAI and DAPSA, respectively. $49.56 \%$ patients presented an elevation of acute phase reactants. Vitamin $D$ levels were 23.81 (+/- 10.5 SD). $77.4 \%$ presented figures of vitamin D deficiency or insufficiency. When performing the association analysis, the vitamin $D$ deficit / insufficiency presented an OR $10(95 \% \mathrm{Cl}$ : 3.66-27.29, $\mathrm{p}=<0.0001)$ with the degree of activity measured with BASDAI and DAPSA and against the elevation of RCP it was $3.63(95 \% \mathrm{Cl} 1.43-9.25, \mathrm{p}=0.0092)$ and against the elevation of ESR it was $2.76(95 \% \mathrm{Cl} 1.09-7,0, \mathrm{p}=0.0438)$. Regarding the comparative analysis of means between vitamin D deficiency/insufficiency and BASDAI/DAPSA it was +3.29 (95\% Cl: 1.34-8.09, $\mathrm{p}=0.0084)$.

Conclusion: Patients with spondyloarthritis, as in other autoimmune diseases, vitamin $D$ deficiency is associated with increased inflammatory activity (BASDAI, DAPSA, RCP and ESR), measured in different time periods. Therefore, an optimization of vitamin $D$ levels can imply an improvement in the patient's clinical situation, measured by both BASDAI and DAPSA, as well as by RCP and ESR. In addition, it is necessary to monitor bone mineral density due to the risk of fracture in these patients for their multietiology (corticosteroid treatments, biological FAMEs, inflammatory activity).

\section{References:}

[1] Lange U, Teichmann J, Strunk J, Müller-Ladner U, Schmidt KL. Association of 1.25 vitamin D3 deficiency, disease activity and low bone mass in ankylosing spondylitis. Osteoporos Int. 2005;16:1999-2004.

[2] Durmus B, Altay Z, Baysal O, Ersoy Y. Does vitamin D affect disease severity in patients with ankylosing spondylitis? Chin Med J. 2012;125:2511-2515.

[3] Mermerci Baskan B, Pekin Dogan y, Sivas F, Bodur H, Ozoran K. The relation between osteoporosis and vitamin $\mathrm{D}$ levels and disease activity in ankylosing spondylitis. Rheumatol Int. 2010;30:375-381.

Disclosure of Interests: None declared

DOI: 10.1136/annrheumdis-2020-eular.1637

\section{AB1282-HPR CONCORDANCE BETWEEN TUBERCULIN TEST AND INTERFERON-GAMMA RELEASE ASSAY IN THE SCREENING OF LATENT TUBERCULOSIS INFECTION IN PATIENTS WHO ARE GOING TO INITIATE A TNF INHIBITOR}

M. D. M. Cutillas Perez ${ }^{1}$, C. Marin Silvente ${ }^{1}$, E. Saiz ${ }^{1}$, M. F. Pina ${ }^{1} .{ }^{1}$ Meseguer General University Hospital Morales, Murcia, Spain

Background: The drugs that inhibit tumor necrosis factor (anti-TNF) alpha can reactivate a latent tuberculosis infection (ILTB) so requiring a rigorous screening before its onset. The tuberculin test (PT) has a high false negative rate in patients with immunomediated rheumatic diseases (IMID) and false positive in patients vaccinated with Bacillus Calmette Guérin (BCG). The neu methods of interferon gamma release (IGRA) seem to solve this problem, but its use is not standardized.

Objectives: Establish the degree of concordance in the diagnosis of ILTB between PT and IGRA in patients who are going to star an anti-TNF drug, in general, and in different situation like taking corticosteroids, being treated with disease modifying drugs, have been vaccinated with BCG or have risk factor for ILTB.

Methods: From May 2016 to November 2019, 195 patients with IMID who underwent ITLB screening prior to the initiation of an anti-TNF drug were included in this study. The concordance between PT and IGRA was calculated using the cohen's kappa index, for the general sample first and then for subgroups. An analysis of the factor that influence the result of PT and IGRA has also been carried out. 